\title{
Non spontaneous saccadic movements identification in clinical electrooculography using machine learning
}

\author{
Roberto Becerra-Garía ${ }^{1}$, Rodolfo García-Bermúdez ${ }^{3}$, Gonzalo Joya-Caparrós ${ }^{2}$, \\ Abel Fernández-Higuera ${ }^{1}$, Camilo Velázquez-Rodríguez ${ }^{1}$, Michel \\ Velázquez-Mariño ${ }^{1}$, Franger Cuevas-Beltrán ${ }^{1}$, Francisco Garcia-Lagos ${ }^{2}$, and \\ Roberto Rodríguez-Labrada ${ }^{4}$ \\ 1 Universidad de Holguín, Grupo de Procesamiento de Datos Biomédicos (GPDB), \\ Holguín, Cuba \\ idertator, afernandezh, cvelazquezr, mvelazquez@f acinf .uho.edu.cu, \\ fcuevas@facii.uho.edu.cu \\ 2 Universidad de Málaga, Málaga, España \\ gjoya@uma.es, lagos@dte.uma.es \\ 3 Universidad Laica Eloy Alfaro de Manabí, Facultad de Informática, Manta, \\ Ecuador \\ rodolfo.garcia@live.uleam.edu.ec \\ ${ }^{4}$ Centro para la Investigación y Rehabilitación de las Ataxias Hereditarias, Holguín, \\ Cuba \\ roberto@ataxia.hlg.sld.cu
}

\begin{abstract}
In this paper we evaluate the use of the machine learning algorithms Support Vector Machines, K-Nearest Neighbors, CART decision trees and Naive Bayes to identify non spontaneous saccades in clinical electrooculography tests. Our approach tries to solve problems like the use of manually established thresholds present in classical methods like identification by velocity threshold (I-VT) or identification by dispersion threshold (I-DT). We propose a modification to an adaptive threshold estimation algorithm for detecting signal impulses without the need of any user input. Also, a set of features were selected to take advantage of intrinsic characteristics of clinical electrooculography tests. The models were evaluated with signals recorded to subjects affected by Spinocerebellar Ataxia type 2 (SCA2). Results obtained by the algorithm shows accuracies over $97 \%$, recalls over $97 \%$ and precisions over $91 \%$ for the four models evaluated.
\end{abstract}

Keywords: Saccade identification, clinical electrooculography, classification

\section{Introduction}

The alteration of eye movements is one of the symptoms of many neurological diseases like Parkinsons syndrome, spinocerebellar ataxias or the Niemann-Pick 
disease [4]. Specifically in the Spinocerebellar Ataxia type 2 (SCA2) this alteration is an important clinical marker present in more than $90 \%$ of patients [29].

There are several kind of eye movements such as saccades, fixations and pursuits. Among them, saccades are critical to follow and evaluate subjects with SCA2. For instance, SCA2 patients have significantly slower saccades and with larger latencies than healthy subjects [29]. The analysis of this kind of movement is used very often in the researches conducted by medical community, hence its importance.

A technique for measuring eye movements called electrooculography consists in capturing the electrical potential of the eyes to calculate its magnitude and direction. This technique is widely used in electrophysiologic tests [16]. The resulting signals of this recording process are named electrooculograms [6].

Exists several methods and algorithms for identifying saccades in electrooculograms, the vast majority of them based on kinetic thresholds $[11,14,31,26]$, using suppervised learning $[28,6]$, unsupervised learning [20] or other novel approachs $[18,22]$ like particle filters [8]. These methods were designed to work in a not constrained scheme having advantages in a lot of scenarios. They are usually evaluated against data from healthy subjects where the differences between saccadic and non saccadic movements are very evident. However, in electrooculography clinical tests these methods try to detect as many saccades as posible, not distinguishing which of them are spontaneous and which not.

In a previous work [2], we proposed a method that identifies saccadic movements using a sample-to-sample approach. This method allows us to discriminate where a sample belong to a saccadic movement or not. Now, in this work we have the task to identify which of these movements are stimuli related using a feature-based approach.

Here we set out to evaluate the use of machine learning algorithms taking into account the strengths of clinical tests of electrooculography to solve the proposed task. Our approach have to use only horizontal movement signals and stimulus signals, and do not require the use of thresholds or any other user input. To do so, a new set of features were selected for training the models taking into account characteristics of valid saccadic movements.

To identify the ocurrence of saccadic movements we use an impulse detection method based on velocity thresholds. These thresholds are calculated adaptively with a modified version of the method proposed by Nyström and Holmqvist [18]. Our algorithm uses a classification model for solving the presented task, so we evaluate four of them: Support Vector Machines [7], K-Nearest Neighbors [27], CART decision tree [5] and Naive Bayes [25]. The performance of the classification models were measured, obtaining very good results ( $>97 \%$ accuracy) in all of them.

The rest of this paper is organized as follows: In section 2 we describe the designed experiments and available data. Section 3 is devoted to analize and comment the results. Finally, section 4 summarizes the main conclusions and future work lines. 


\section{Material and Methods}

To test the selected algorithms an experiment was designed. The first step was identify potential impulses and annotating them for building a labeled dataset. After, each classification method is evaluated with stratified $k$-fold cross validation. Finally, we compare the performance of the models using nonparametric statistical tests to select the fittest.

Clinical tests of electrooculography are setup as follows. Subjects with their head fixed are seated in front to a monitor at a previously known distance. Then, they are commanded to follow a visual stimuli which appears and disappears from one side to the other in the monitor. Capturing eye movements in these conditions using electrooculography allows to researchers the identification of which saccades respond to stimulus and which ones are spontaneous. Also allows to calculate important features of these movements like latency, duration, amplitude, deviation and maximal velocity.

The electrooculograms were recorded using the OtoScreen electronystamography device at a sampling rate of $200 \mathrm{~Hz}$ with a bandwith of 0.02 to $70 \mathrm{~Hz}$. Records of 12 sick subjects with SCA2 were used for building a dataset with features extracted from signal impulses. Each one of the records have at least tests of $10^{\circ}, 20^{\circ}$ and $30^{\circ}$ of visual stimulation. Typically saccadic tests have at least one horizontal channel and one stimulus signal (Fig. 1).
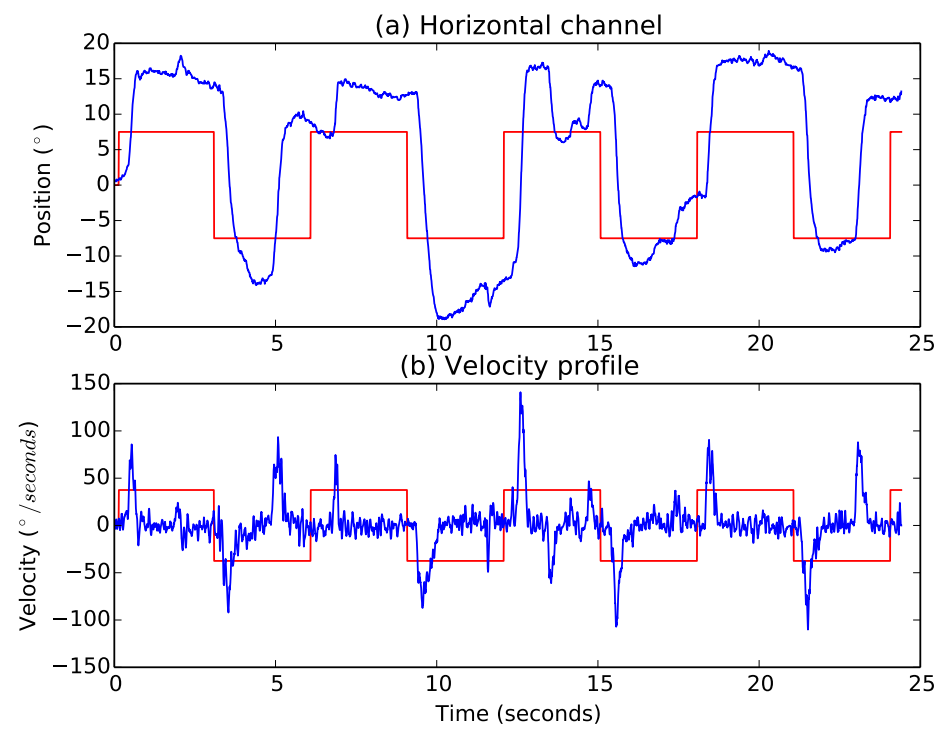

Fig. 1. Typical electrooculography signal with $30^{\circ}$ stimulus angle of a subject which suffers SCA2. Red signals are the scaled stimuli signals. Blue signals are the horizontal channel (a) and its velocity profile (b) respectively. 
IPython notebooks [23] were used in conjunction with the Python language scientific facilities: NumPy [19], SciPy [12], Pandas [17], Matplotlib [10] and Scikit-Learn [21] for running the experiments. These notebooks provides a rich interactive environment wich eases the development of experiments and facilitates the interchange of information between the authors. The intention behind using Python powered technologies is that the resulting algorithm (including trained models) will be used at NSEog, a processing platform developed by the authors.

\subsection{Signals preprocessing}

Before the identification of potentially saccadic impulses, two common tasks needs to be performed: denoising and differentiation. Noise removal is very important matter in order to eliminate non desired spectral components due equipment malfunction, poor analog filtering or biological artifacts. Differentiation allows to obtain the velocity profile used later by the algorithm.

Median filter (Equation 1) has proven to be very robust in eliminating high frequency signal noise while preserving sharp edges. An study carried out by Juhola in 1990 demonstrated that this kind of filters is appropiate for eye movements signals [13]. To eliminate non desired noise present in the signals used in the experiment, we use a median filter with a window size of 9 samples (approximately 45 milliseconds) obtaining very good results. This is accomplished using the medfilt function of SciPy.

$$
y_{i}=\operatorname{median}\left\{x_{j} \mid j=i-k, \ldots, j+k\right\}
$$

Due the discrete nature of these signals, numerical differentiation is employed to calculate the velocity profiles. According [3], Lanczos differentiators (Equation $2)$ with 11 points $(N=11)$ have good performance for signals with the same characteristics as the ones used in this experiment.

$$
f^{\prime}\left(x^{*}\right) \approx \frac{3}{h} \sum_{k=1}^{m} k \frac{f_{k}-f_{-k}}{m(m+1)(2 m+1)}, \quad m=\frac{N-1}{2}
$$

We implemented the rutine of a Lanczos 11 differentiator which have the following formula:

$$
f^{\prime}\left(x^{*}\right) \approx \frac{f_{1}-f_{-1}+2\left(f_{2}-f_{-2}\right)+3\left(f_{3}-f_{-3}\right)+4\left(f_{4}-f_{-4}\right)+5\left(f_{5}-f_{-5}\right)}{110 h}
$$

\subsection{Impulses detection}

Saccadic movements are represented as impulses in a velocity graph as shown in Fig. 1b. Typically, this movements can be easily identified by its contrast in magnitude and shape with other movements such as fixations and microsaccades. 
However, for the same stimulus angle the range of values of true saccadic impulses vary from subject to subject. This situation is tied greatly on the degree of affectation present in the subject [24].

One of the critical parts of the algorithm is the identification of velocities impulses which can potentially be saccades. For that matter, a threshold is needed for knowing when the velocity has reached a certain value that can be considered as a saccade candidate. Due the inter-subject variability explained before, this threshold should not be fixed a priori. Also should be large enough to ignore in most cases other movements like microsaccades and fixations, and not too large to miss valid saccadic movements.

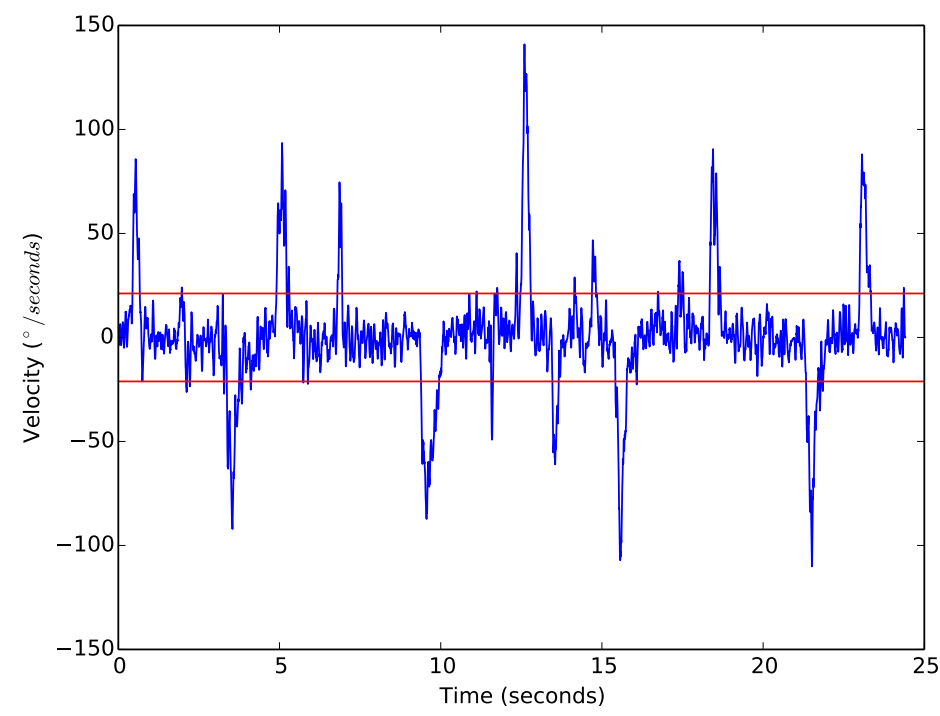

Fig. 2. Threshold estimated in a $30^{\circ}$ stimulus angle test of a subject with SCA2.

Estimation of a good velocity threshold is not an easy task, depending also on the noise levels present in the signal. The method described in Algorithm 1 solved this problem by calculating the threshold in an adaptive way. This is possible by using a safety margin $(\sigma)$ based on the standard deviation of the signal which get adapted to the noise level present in the signal.

The safety margin $(\sigma=6)$ employed by [18] ignores too many valid saccadic movements in lower angle tests for subjects with SCA2. A value of $\sigma=3$ seems to be adequate for most cases at the expense of the detection of more non valid impulses. Even when this have a penalty in runtime performance, the final accuracy of the method should not decrease significantly. Due the amplitude of this new impulses the classification model should avoid them. 


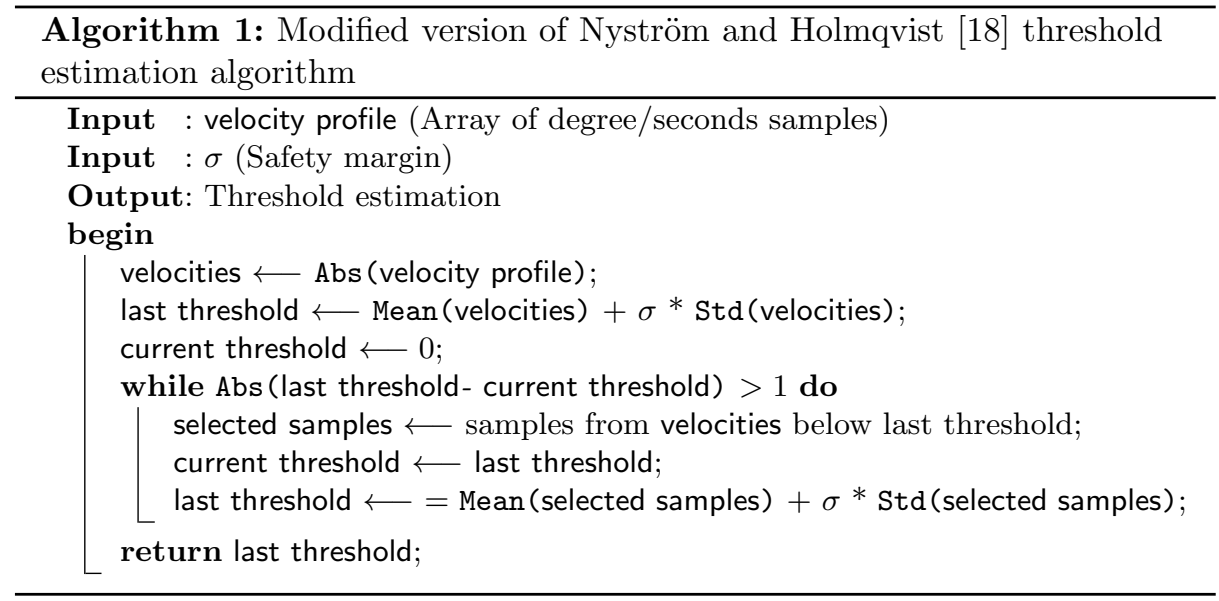

Another problem with this method [18] is that requires an initial threshold. This adds a bit of subjectivity to the overall process because this value could vary from healthy subjects to affected ones. We introduce a little modification to the method which states that this initial threshold is calculated the same way as the thresholds inside the iterations in the Algorithm 1. The only difference is that our method uses all velocity samples to calculate the mean and the standard deviation. This approach seems to work very well for all analyzed tests. Once the threshold is calculated, the next task is found all the impulses above it. This is accomplished by finding samples grouped together that exceeds this threshold.

The principle behind this algorithm is looping through the signal to find velocities above the given threshold. When we encounter with one of these points, we move to the left and to the right until the velocity is zero or cross it. This approach allows further refinement of the saccade start and ending points because the impulses usually get more samples beyond the real saccade limits. If the length of a detected impulse is not greater than 10 samples, then is discarded to avoid very small invalid movements. A typical output of this method is represented in Fig. 3.

\subsection{Model evaluation}

Once identified the saccadic impulses candidates, we need to know if they are saccades and if they are related to the stimulus. For this reason, the strategy behind our approach uses human intuitive features to solve this task. To take advantage of the characteristics of the clinical tests, the following set of features was carefully selected:

Angle: Integer denoting the amplitude of the stimulus which can take 3 values: 10,20 or 30 .

Absolute Latency: Time between the start of the stimulus transition and the maximal velocity point of the impulse in milliseconds (ms). 


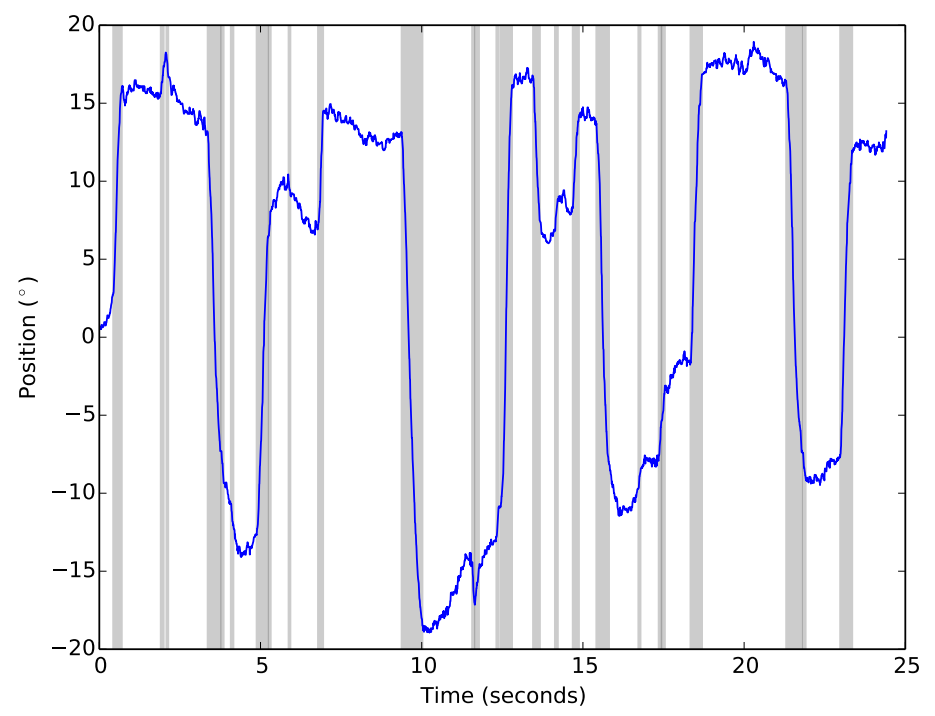

Fig. 3. Identified impulses in the same signal used in Figure 2

Normalized Latency: Normalized version of absolute latency with values between 0 and 1 . The value 0 means that the maximal velocity is in the start of the stimulus transition, and the value 1 means that the maximal velocity is at the end of the stimulus transition.

Amplitude: Difference between the maximum value and minimum value in the impulse.

Deviation: Relation between amplitude and the angle of the stimulus.

Maximum Velocity: Maximum velocity achieved during the impulse in $\%$.

Maximum Acceleration: Maximum acceleration achieved during the impulse in $\% / s^{2}$.

Maximum Jerk: Maximum jerk achieved during the impulse in $\% / s^{3}$.

Direction: Take the value 1 if the impulse follows the direction of the stimulus or -1 in other case.

End Relative Position: Values between 0 and 1 . The value 0 means that the impulse ends in the left position of the stimulus and the value 1 means that ends at the right of the stimulus.

Using the features previously selected, a dataset of signal impulses was created. For building this dataset, a human specialist aided by the NSEog classified the detected impulses in valid and non valid saccades (Figure 4). As results, 1797 valid saccades and 6809 not valid impulses were obtained, resulting in 8606 instances.

Because we are using Python technologies, Scikit-Learn was selected as machine learning library, hence we are constrained to a restricted set of models im- 


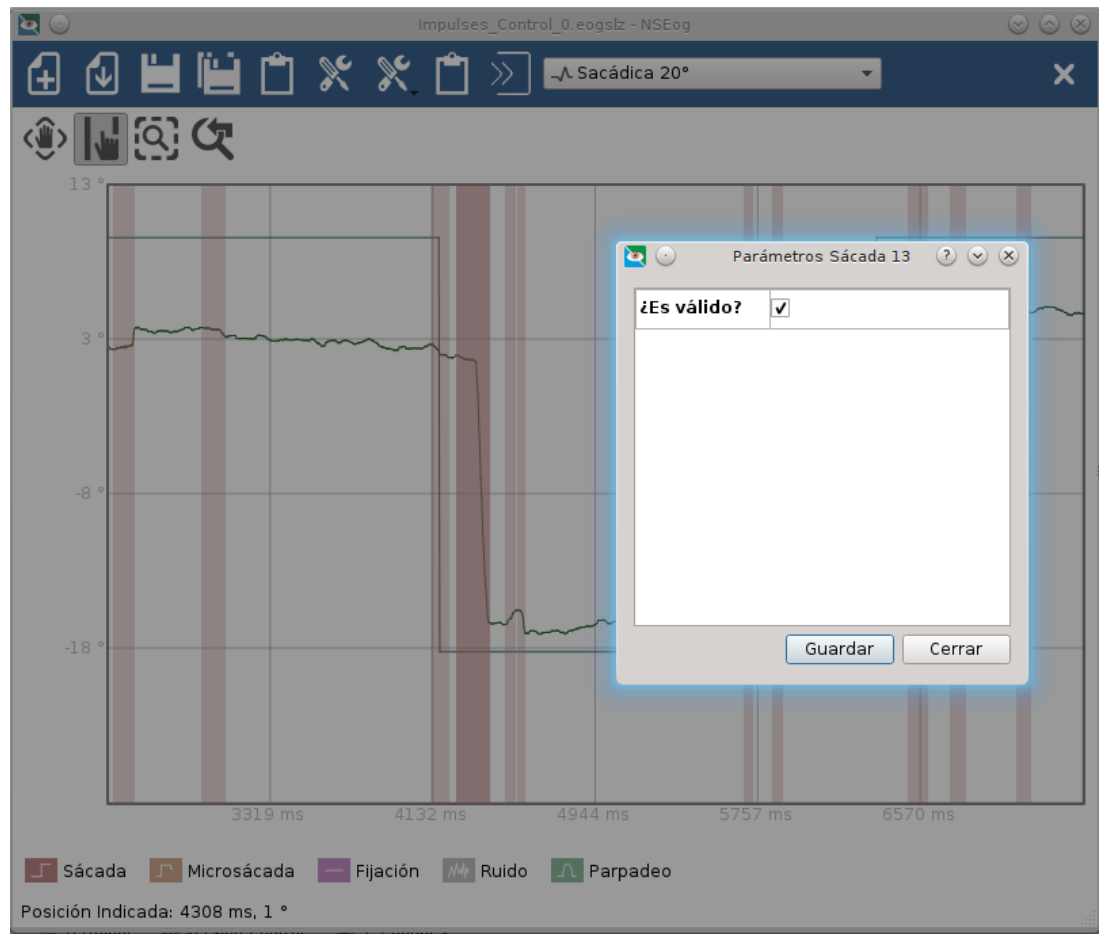

Fig. 4. Impulses annotation with the NSEog platform. 
plemented in it. The main policy of model selection was family representation, meaning that we try to choose methods with different working principles. So we evaluate four different models: Support Vector Machines, K-Nearest Neighbors, CART decision trees and the Gaussian version of Naive Bayes.

Support Vector Machines (SVMs) are a set of supervised learning methods very effective in high dimensional spaces [7]. There are also very versatile supporting a set of kernel functions. Scikit-Learn implements four kernel functions: linear $\left\langle x, x^{\prime}\right\rangle$, polynomial $\left(\gamma\left\langle x, x^{\prime}\right\rangle+r\right)^{d}$, rbf $e^{-\gamma\left|x-x^{\prime}\right|^{2}}$ and sigmoid $\tanh \left(\gamma\left\langle x, x^{\prime}\right\rangle+r\right)$. Preliminary experiments shows that for the proposed task, the rbf kernel function have the best performance compared with the others. Further study are necessary to fine tune the parameter $\gamma$ of this kernel.

K-Nearest Neighbors is a type of instance-based learning which can be used for supervised or unsupervised learning. Instead of creating a generalizing function, it stores all the data inside the models using different data structures like Ball Trees or KD Trees. The principle behind the algorithm is find a number of training samples nearest to the analized point and predict the label from it [27]. To train our model we tried several numbers of neighbors starting from 2, giving the best results when this value is equal to 3 . The data structure used is determined automatically by the Scikit-Learn implementation using optimization techniques.

Decision trees are nonparametric supervised learning techniques. This algorithm requires little preprocessing and its runtime performance is enough to handle real time tasks. This method split the data trying to infere decision rules which can be used to clasify instances. Scikit-Learn uses an optimized version of the CART tree whichs support classification and regression [5]. The implementation used here do not require any parameter by default.

Naive Bayes classifiers are supervised methods based on Bayes theorem which assumes independence between every pair of features [25]. We used a gaussian version of this classifier implemented in Scikit-Learn. Like the decision trees, the default implementation of this statistical classifier do not require any parameters.

As validation scheme we use an stratified 10 -fold cross validation to evaluate internally the models. The metrics employed to measure the performance were accuracy (Equation 4), recall (Equation 5) and precision (Equation 6) [30]. The accuracy give us a general quality measure of the performance of the models, while the recall and the precision allows to know how well the model predict or miss predict valid saccadic movements. In the following equations, TP (true positives), TN (true negatives), FP (false positives) and FN (false negatives) are the items from the confusion matrix used to compute involved metrics.

$$
\begin{gathered}
\text { Accuracy }=\frac{T P+T N}{T P+F P+T N+F N} \\
\text { Recall }=\frac{T P}{T P+F N} \\
\text { Precision }=\frac{T P}{T P+F P}
\end{gathered}
$$


The whole dataset was adjusted by removing the mean and scale to unit variance. This technique is critical for obtaining good results in the training of the RBF kernel version of Support Vector Machines. These scales was saved along with the model for further use by the algorithm.

To compare the real performance of the models, the Friedman's nonparametric statistical test were used as recommended by Demšar [9]. In this step we use records not used previously for training the models. Each metric were analyzed by separate and the statistical calculations were performed using the Keel tool $[1]$.

The resulting classification algorithm is very simple and flexible. It consists in the evaluation of the features calculated from impulses detected in the signal by the supervised model. This approach allows the parallelization of the algorithm and even swap the model if needed. Due the use of the proposed impulse detection algorithm, the need for parameters managed by the user is eliminated.

\section{Results}

The evaluated models were trained with 8606 impulses, 1797 valid saccades and 6809 invalid ones. Using 10-fold cross validation the internal performance of the trained process was measured with the metrics accuracy, recall and precision. Table 1 shows results above .97 of accuracy, .94 of recall and .90 of precision in all cases.

Table 1. 10-fold cross validation results

\begin{tabular}{lccc}
\hline Model & Acc. & Rec. & Pre. \\
\hline$S V M$ & 0.9833 & 0.9750 & 0.9467 \\
KNN & 0.9796 & 0.9666 & 0.9376 \\
CART & 0.9769 & 0.9449 & 0.9445 \\
NaiveBayes & 0.9747 & 0.9817 & 0.9056 \\
\hline
\end{tabular}

To perform a more objective evaluation, the algorithm was tested against five new subject records not used in the training phase. A total of 3797 impulses were evaluated this time, 704 real saccadic impulses and 3093 not saccadic.

Table 2. External validation results by stimulus amplitude

\begin{tabular}{|c|c|c|}
\hline & KNN & NaiveBayes \\
\hline & Rec. $\mathbf{P}$ & c. Pre. Acc \\
\hline 10 & .9765 .9703 .9051 .9659 .9449 .8745 & .9636 .9237 .8790 .9575 .9661 .8261 \\
\hline 20 & 844.9837 .9305 & 8993.9837. 9780. 9636.9365. \\
\hline 30 & 8745. 9620.9686.9038.9646. & $\begin{array}{llll}.9674 .9462 .9017 .9543 & 9686 & .9372\end{array}$ \\
\hline Mean & .9780 .9742 .9155 .9716 .9657 .8932 & .9711 .9444 .9058 .9633 .9728 .8542 \\
\hline Std & 0323. 0195. 0111. 0192. 0082. 0070. & 0394. 0095. 0128. 0290. 0198. 0098. \\
\hline
\end{tabular}


Results obtained analysing the performance individually by stimulus angle seems to favor lightly the SVM model (Table 2). However, doing the same analysis using independent subject records shows a more erratic behaviour (Table 3 ). Because of this situation, the Friedman's nonparametric statistical test was employed to compare the performance of the four models. Each record was considered as an individual dataset and each of the three performance metrics was analyzed independently using the data in Table 3. Results obtained by this method shows that there are no significant differences in the performance of these models for a significance level of $p=0.10$.

Table 3. External validation results by subject record

\begin{tabular}{|c|c|c|c|}
\hline & SVM & CART & NaiveBayes \\
\hline Subject & Rec. Pre. & Acc. Rec. Pre. Acc. Rec. Pre & cc. Rec. Pre. \\
\hline 1 & .9881 .9877 .9699 & .9881.9877.9699.9796.9693.9576 & .9881 .9755 .9815 \\
\hline 2 & .9862.9935.9107 & 9048. 9870. 9845. 8506. 9610. & .9535.9935.7427 \\
\hline 3 & .9871 .9754 .9444 & .9794.9590.9141.9704. 8852. & .9717 .9836 .8571 \\
\hline 4 & .9799 .9420 .9559 & 9545.9784.9348.9556.9741. & .9756 .9420 .9353 \\
\hline 5 & .9410 .9685 .8039 & 7961. 9392.9843. 7911. 9358. & .9375 .9685 .7935 \\
\hline Mean & .9765 .9734 .9170 & .9715.9654.8962.9689.9415.9072 & .9653 .9726 .8620 \\
\hline Std & .0201.0201.0669 & 0659. 0417. 0193. 0748. 0215 & .0199.0195.0982 \\
\hline
\end{tabular}

Literature about the task proposed in this work are scarce and no methods to solve it specifically were founded. However, similar works reported a recall of .89 for $10^{\circ}$ recordings on healthy subjects [22] and .80 of recall on subjects with Obstructive Sleep Apnea Syndrome (OSAS) [15]. Other related research conducted by Tigges et al. shows an accuracy of .92 [28]. Taking into account that we are dealing with signals recorded to subjects which suffers a very severe neurological disorder, results shown in Table 2 and Table 3 are better than the others presented in the literature.

\section{Conclusions}

We have described a procedure to identify non spontaneous saccades which uses an impulses detection algorithm and supervised classification models. This procedure do not requires thresholds or any other user input and uses a feature-based classification approach. The application of this algorithm in signals recorded from patients with SCA2 shows high performance values.

The four evaluated learning models are suitable to be used in the algorithm without significant performance differences. This performance is at least equal or greater than similar works founded in the literature. Further study is required to establish accurately the onset and offset points of valid saccadic impulses. 


\section{Acknowledgements}

We'd like to thank the undergraduate students of the Grupo de Procesamiento de Datos Biomédicos for their support in the development of this paper, specifically to Fidel Antonio Cabrera Bruzón and Pedro Enrique Almirall Abreu.

\section{References}

1. Alcalá-Fdez, J., Sánchez, L., García, S., del Jesús, M.J., Ventura, S., Garrell, J.M., Otero, J., Romero, C., Bacardit, J., Rivas, V.M., others: KEEL: a software tool to assess evolutionary algorithms for data mining problems. Soft Computing 13(3), 307-318 (2009), http://link.springer.com/article/10.1007/s00500-008-0323-y

2. Becerra, R., Joya, G., Bermúdez, R.V.G., Velázquez, L., Rodríguez, R., Pino, C.: Saccadic points classification using multilayer perceptron and random forest classifiers in eog recordings of patients with ataxia sca2. In: Rojas, I., Joya, G., Cabestany, J. (eds.) Advances in Computational Intelligence, pp. 115-123. Springer Berlin Heidelberg (2013)

3. Becerra García, R.A.: Plataforma de procesamiento de electrooculogramas. Caso de estudio: pacientes con Ataxia Espinocerebelosa Tipo 2. Master en matemática aplicada e informática para la administración, Universidad de Holguín, Holguín (2013)

4. Bonnet, C., Hanuška, J., Rusz, J., Rivaud-Péchoux, S., Sieger, T., Majerová, V., Serranová, T., Gaymard, B., Růžička, E.: Horizontal and vertical eye movement metrics: What is important? Clinical Neurophysiology 124(11), 2216-2229 (Nov 2013), http://www.clinph-journal.com/article/S1388245713006378/abstract

5. Breiman, L., Friedman, J., Stone, C.J., Olshen, R.A.: Classification and regression trees. CRC press (1984)

6. Bulling, A., Ward, J., Gellersen, H., Troster, G.: Eye Movement Analysis for Activity Recognition Using Electrooculography. IEEE Transactions on Pattern Analysis and Machine Intelligence 33(4), 741-753 (2011)

7. Cortes, C., Vapnik, V.: Support-vector networks. Machine Learning 20(3), 273-297 (Sep 1995), http://link.springer.com/article/10.1007/BF00994018

8. Daye, P.M., Optican, L.M.: Saccade detection using a particle filter. Journal of neuroscience methods 235, 157-168 (2014)

9. Demšar, J.: Statistical comparisons of classifiers over multiple data sets. The Journal of Machine Learning Research 7, 1-30 (2006), http://dl.acm.org/citation.cfm?id=1248548

10. Hunter, J.D.: Matplotlib: A 2D Graphics Environment. Computing in Science and Engineering 9(3), 90-95 (2007)

11. Inchingolo, P., Spanio, M.: On the Identification and Analysis of Saccadic Eye Movements-A Quantitative Study of the Processing Procedures. IEEE Transactions on Biomedical Engineering BME-32(9), 683-695 (1985)

12. Jones, E., Oliphant, T., Peterson, P.: SciPy: Open source scientific tools for Python. http://www. scipy. org/ (2001), http://www.citeulike.org/group/2018/article/2644428

13. Juhola, M.: Median filtering is appropriate to signals of saccadic eye movements. Computers in Biology and Medicine 21(1-2), 43-49 (1991), http://www.sciencedirect.com/science/article/pii/0010482591900347 
14. Juhola, M., Jäntti, V., Pyykkö, I., Magnusson, M., Schalén, L., Åkesson, M.: Detection of saccadic eye movements using a non-recursive adaptive digital filter. Computer Methods and Programs in Biomedicine 21(2), 81-88 (Nov 1985), http://www.sciencedirect.com/science/article/pii/0169260785900665

15. Magosso, E., Provini, F., Montagna, P., Ursino, M.: A wavelet based method for automatic detection of slow eye movements: A pilot study. Medical Engineering \& Physics 28(9), 860-875 (Nov 2006), http://www.sciencedirect.com/science/article/pii/S1350453306000154

16. Marmor, M.F., Zrenner, E.: Standard for clinical electrooculography. Documenta Ophthalmologica 85(2), 115-124 (Feb 1993), http://dx.doi.org/10.1007/BF01371127

17. McKinney, W.: Data Structures for Statistical Computing in Python. pp. 51-56 (2010), http://conference.scipy.org/proceedings/scipy2010/mckinney.html

18. Nyström, M., Holmqvist, K.: An adaptive algorithm for fixation, saccade, and glissade detection in eyetracking data. Behavior Research Methods 42(1), 188-204 (Feb 2010), http://link.springer.com/article/10.3758/BRM.42.1.188

19. Oliphant, T.E.: Python for scientific computing. Computing in Science \& Engineering 9(3), 10-20 (2007), http://scitation.aip.org/content/aip/journal/cise/9/3/10.1109/MCSE.2007.58

20. Otero-Millan, J., Castro, J.L.A., Macknik, S.L., Martinez-Conde, S.: Unsupervised clustering method to detect microsaccades. Journal of vision 14(2), 18 (2014)

21. Pedregosa, F., Varoquaux, G., Gramfort, A., Michel, V., Thirion, B., Grisel, O., Blondel, M., Prettenhofer, P., Weiss, R., Dubourg, V., Vanderplas, J., Passos, A., Cournapeau, D., Brucher, M., Perrot, M., Duchesnay, E.: Scikit-learn: Machine Learning in Python. Journal of Machine Learning Research 12, 2825-2830 (Oct 2011), http://jmlr.csail.mit.edu/papers/v12/pedregosa11a.html

22. Pettersson, K., Jagadeesan, S., Lukander, K., Henelius, A., Hæggström, E., Müller, K.: Algorithm for automatic analysis of electro-oculographic data. Biomedical engineering online 12(1), 110 (2013)

23. Pérez, F., Granger, B.E.: IPython: A System for Interactive Scientific Computing. Computing in Science \& Engineering 9(3), 21-29 (May 2007), http://scitation.aip.org/content/aip/journal/cise/9/3/10.1109/MCSE.2007.53

24. Rodríguez-Labrada, R., Velázquez-Pérez, L.: Eye movement abnormalities in spinocerebellar ataxias. Spinocerebellar ataxias. Rijeka: Intech pp. 59-76 (2012)

25. Russell, S., Norvig, P.: Artificial Intelligence: A Modern Approach. Prentice Hall, Upper Saddle River, NJ, 3 edition edn. (Dec 2009)

26. Salvucci, D.D., Goldberg, J.H.: Identifying fixations and saccades in eyetracking protocols. pp. 71-78. ETRA '00, ACM, New York, NY, USA (2000), http://doi.acm.org/10.1145/355017.355028

27. Silverman, B.W., Jones, M.C.: An Important Contribution to Nonparametric Discriminant Analysis and Density Estimation. International Statistical Review / Revue Internationale de Statistique 57(3), 233-238 (1989), http://www.jstor.org/stable/1403796

28. Tigges, P., Kathmann, N., Engel, R.R.: Identification of input variables for feature based artificial neural networks-saccade detection in EOG recordings. International Journal of Medical Informatics 45(3), 175-184 (Jul 1997), http://www.sciencedirect.com/science/article/pii/S1386505697000427

29. Velázquez-Pérez, L., Rodríguez-Labrada, R., García-Rodríguez, J.C., AlmaguerMederos, L.E., Cruz-Mariño, T., Laffita-Mesa, J.M.: A comprehensive review of spinocerebellar ataxia type 2 in cuba. The Cerebellum 10(2), 184-198 (Jun 2011), http://link.springer.com/article/10.1007/s12311-011-0265-2 
30. Witten, I.H., Frank, E., Hall, M.A.: Data Mining. Practical Machine Learning Tools and Techniques. Morgan Kaufmann, third Edition edn. (2011)

31. Wyatt, H.J.: Detecting saccades with jerk. Vision Research 38(14), 2147-2153 (Jul 1998), http://www.sciencedirect.com/science/article/pii/S0042698997004100 